\title{
Gravity-wave interferometers as probes of a low-energy effective quantum gravity
}

\author{
Giovanni Amelino-Camelia* \\ Theory Division, CERN, CH-1211, Geneva, Switzerland \\ (Received 5 April 1999; published 23 June 2000)
}

\begin{abstract}
The interferometry-based experimental tests of quantum properties of space-time which the author sketched out in a recent short paper are here discussed in a self-contained fashion. In addition to providing detailed derivations of the results already announced in the previous paper, some new results are also derived; in particular, the analysis is extended to a larger class of scenarios for space-time fuzziness. It is argued that these studies could be helpful for the search for a theory describing a first stage of partial unification of gravity and quantum mechanics.
\end{abstract}

PACS number(s): 04.60.- m, 04.80.Nn

\section{INTRODUCTION AND SUMMARY}

Perhaps the most fascinating questions confronting contemporary physics concern the search for the appropriate framework for the unified description of gravitation and quantum mechanics. This search for "quantum gravity" is proving very difficult [1], especially as a result of the scarce experimental information available on the interplay between gravitation and quantum mechanics. However, in recent years there has been a small (but nevertheless encouraging) number of new proposals [2-5] of experiments probing the nature of the interplay between gravitation and quantum mechanics. At the same time the "COW-type" experiments, initiated with the celebrated experiment by Colella, Overhauser and Werner [6], have reached levels of sophistication [7] such that even gravitationally induced quantum phases due to local tides can be detected. In light of these developments there is now growing (although still understandably cautious) hope for data-driven insight into the structure of quantum gravity.

The primary objective of the present article is to provide a careful discussion of the most recent addition to the (still far from numerous) family of quantum-gravity experiments, which this author proposed in the short paper in Ref. [8]. This most recent proposal probes in a rather direct way the properties of space-time, which is of course the most fundamental element of a quantum gravity, by exploiting the remarkable accuracy achievable with advanced modern interferometers, such as the ones used for searches of gravity waves.

In addition to emphasizing the ways in which the experiment proposed in Ref. [8] can contribute to the development of "quantum-gravity phenomenology" [9], in this article I shall also relate the class of observations accessible to modern interferometers to a physical picture of the (necessarily small) way in which quantum gravity might affect phenomena probing space-time at distances significantly larger than the Planck length $L_{p} \sim 10^{-35} \mathrm{~m}$ (but significantly shorter than distance scales probed in ordinary particle physics or gravity experiments). This physical picture is motivated by

\footnotetext{
*Present address: Dipartimento di Fisica, Universitá di Roma "La Sapienza," Piazzale Moro 2, Roma, Italy.
}

the huge gap between the minute Planck length and the distance scales probed in present-day particle physics or gravitational experiments. The size of this gap provides motivation for exploring the possibility that on the way to Plancklength physics a few intermediate steps of partial unification of gravity and quantum mechanics might be required before reaching full unification. Of course, as long as we are lacking direct experimental evidence to the contrary, it is also reasonable to work (as many distinguished colleagues do) on the hypothesis that gravitation and quantum mechanics should merge directly into a fully developed quantum gravity, but in the present article (as in the previous papers [1012]) I shall be concerned with the investigation of the properties that one could demand of a theory suitable for a first stage of partial unification of gravity and quantum mechanics. In particular, I shall review the arguments presented in Refs. [11,12] suggesting that the most significant implications of quantum gravity for low-energy (large-distance) physics might be associated with the structure of the nontrivial "quantum-gravity vacuum." A satisfactory picture of this quantum-gravity vacuum is not available at present, and therefore we must generically characterize it as the appropriate new concept that in quantum gravity takes the place of the ordinary concept of "empty space;" however, it is plausible that some of the arguments by Wheeler, Hawking and others (see, e.g., Refs. $[13,14]$ and references therein), which have attempted to develop an intuitive description of the quantum-gravity vacuum, might have captured at least some of its actual properties.

Other possible elements for the search of a theory suitable for a first stage of partial unification of gravity and quantum mechanics come from studies suggesting that this unification might require a novel relationship between "measuring apparatus" and "system." My intuition on the nature of this new relationship is mostly based on work by Bergmann and Smith [15] and the observations I reported in Refs. [10,12], which took as starting point an analysis by Salecker and Wigner [16].

The intuition emerging from these considerations on a novel relationship between measuring apparatus and system and by a Wheeler-Hawking picture of the quantum-gravity vacuum are not sufficient for the full development of a new formalism describing the first stage of partial unification of gravity and quantum mechanics, but they provide encourage- 
ment for the search of a formalism based on a mechanics not exactly of the type of ordinary quantum mechanics. Moreover, one can use this emerging intuition for rough estimates of certain candidate quantum-gravity effects. The estimates most relevant for the present article are the ones concerning the space-time "fuzziness" which modern interferometers could investigate following Ref. [8].

A prediction of nearly all approaches to the unification of gravity and quantum mechanics is that at very short distances the sharp classical concept of space-time should give way to a somewhat "fuzzy" (or "foamy") picture (see, e.g., Refs. $[13,14,17,18])$, but it is usually very hard to characterize this fuzziness in physical operative terms. In Sec. II I provide an operative definition of fuzzy distance, which uses the properties of interferometers. I also introduce a rather general parametrization of distance fuzziness, which could be useful in the analysis or interpretation of experimental results. Section III is devoted to some heuristic arguments which can be used to estimate the nature and magnitude of the quantum fluctuations that might affect distances if space-time hosts an intrinsic mechanism for decoherence. I shall search for plausible (but admittedly "optimistic") estimates of the relevant quantum-gravity effects, and, although quantitative estimates will be derived, the true emphasis is on the qualitative aspects of the phenomena, since this type of information could be helpful to colleagues on the experimental side in establishing how to look for these phenomena. Some of the estimates I provide are motivated by studies of the measurability of distances in quantum gravity. A second group of estimates is motivated by arguments of "consistency" (in the sense discussed later) with recent proposals $[4,19,20]$ of quantumgravity induced deformation of the dispersion relation that characterizes the propagation of massless particles. All of these arguments indicate that a priority for interferometrybased tests of space-time fuzziness must be high sensitivity at low frequencies, and I hope this will be taken into account in planning future interferometers.

In Sec. IV I shall observe (extending the related observations reported in Ref. [8]) that the remarkable sensitivity achieved by modern interferometers, the ones primarily searching for gravity waves [21-25], places significant constraints on the nature and magnitude of distance fuzziness. Perhaps the most intuitive way to characterize the obtained bounds is given by the fact that we are now in a position to rule out a picture of fuzzy space-time such that the 40-m arms of the interferometer considered in Ref. [22] would be affected by minute Planck-length fluctuations occurring at a rate of one per each Planck time $\left(t_{p}=L_{p} / c \sim 3 \times 10^{-44} \mathrm{~s}\right)$. In $\mathrm{Sec}$. V I discuss the aspects of certain existing quantum gravity approaches which are in one or another way related to the type of fuzzy space-times considered in Sec. II. In Sec. VI I discuss how the class of experiments proposed in Ref. [8] (and here analyzed in detail) complements other proposals of quantum gravity experiments. I also outline the general features that an experiment must have in order to uncover aspects of the interplay between gravitation and quantum mechanics. In Sec. VII I use the results discussed in Secs. II-V to better define the idea of a theory appropriate for the description of a first stage of partial unification of gravity and quantum mechanics. Closing remarks, also on the outlook of quantum-gravity phenomenology, are given in Sec. VIII.

\section{PRELIMINARIES ON DISTANCE FUZZINESS}

\section{A. Operative definition of fuzzy distance}

While nearly all approaches to the unification of gravity and quantum mechanics appear to lead to a somewhat fuzzy picture of space-time, within the various formalisms it is often difficult to characterize physically this fuzziness. Rather than starting from formalism, I shall advocate an operative definition of fuzzy space-time. ${ }^{1}$ More precisely for the time being I shall just consider the concept of fuzzy distance. I shall be guided by the expectation that at very short distances the sharp classical concept of distance should give way to a somewhat fuzzy distance. Since interferometers are ideally suited to monitor the distance between test masses, I choose as operative definition of quantum-gravity induced fuzziness one which is expressed in terms of quantum-gravity induced noise in the read-out of interferometers.

In order to articulate this proposal it will prove useful to briefly review some aspects of the physics of Michelson interferometers. These are schematically composed [21] of a (laser) light source, a beam splitter and two fully-reflecting mirrors placed at a distance $L$ from the beam splitter in orthogonal directions. The light beam is decomposed by the beam splitter into a transmitted beam directed toward one of the mirrors and a reflected beam directed toward the other mirror; the beams are then reflected by the mirrors back toward the beam splitter, where [21] they are superposed. ${ }^{2}$ The resulting interference pattern is extremely sensitive to changes in the positions of the mirrors relative to the beam splitter. The achievable sensitivity is so high that planned interferometers $[23,24]$ with arm lengths $L$ of 3 or $4 \mathrm{Km}$ expect to detect gravity waves of amplitude $h$ as low as $3 \times 10^{-22}$ at frequencies of about $100 \mathrm{~Hz}$. This roughly means that these modern gravity-wave interferometers should monitor the (relative) positions of their test masses (the beam splitter and the mirrors) with an accuracy [22] of order $10^{-18} \mathrm{~m}$ and better.

\footnotetext{
${ }^{1}$ Once we have a physical definition of fuzzy space-time the analysis of the various quantum-gravity formalisms could be aimed at providing predictions for this fuzziness. Of course, in order for the formalisms to provide such physical predictions it is necessary to equip them with at least some elements of a "measurement theory."

${ }^{2}$ Although all modern interferometers rely on the technique of folded interferometer's arms (the light beam bounces several times between the beam splitter and the mirrors before superposition), I shall just discuss the simpler "no-folding" conceptual setup. The readers familiar with the subject can easily realize that the observations here reported also apply to more realistic setups, although in some steps of the derivations the length $L$ would have to be understood as the optical length (given by the actual length of the arms times the number of foldings).
} 
In achieving this remarkable accuracy experimentalists must deal with classical-physics displacement noise sources (e.g., thermal and seismic effects induce fluctuations in the relative positions of the test masses) and displacement noise sources associated with effects of ordinary quantum mechanics (the combined minimization of photon shot noise and radiation pressure noise leads to an irreducible noise source which has its root in ordinary quantum mechanics). The operative definition of fuzzy distance which I advocate characterizes the corresponding quantum-gravity effects as an additional source of displacement noise. A theory in which the concept of distance is fundamentally fuzzy in this operative sense would be such that the read-out of an interferometer would still be noisy (because of quantum-gravity effects) even in the idealized limit in which all classical-physics and ordinary quantum-mechanics noise sources are completely eliminated.

Adopting this operative definition of fuzzy distance, interferometers are of course the natural tools for experimental tests of proposed space-time fuzziness scenarios. However, even the remarkable sensitivity estimate of order $10^{-18} \mathrm{~m}$ given above is quite far from the Planck length $\sim 10^{-35} \mathrm{~m}$, and it might appear safe to assume that any scenario for space-time fuzziness would not observably affect the operation of even the most sophisticated modern interferometers. As I shall discuss in greater detail later in this article, this scale comparison is not the correct measure of the sensitivity of an interferometer to Planck-length fluctuations. Depending on the nature of the conjectured space-time stochastic processes, the interferometer might be (and it is) sensitive to the collective effect of a large number of fluctuations. The proper way to compare a given model of distance fluctuations and the sensitivity of a given interferometer is through the amplitude spectral density of predicted distance fluctuations on one side and the amplitude spectral density of observed distance fluctuations ("noise") on the other side. We therefore need at least a rough idea of which types of amplitude spectral density of distance fluctuations could be predicted by quantum gravity. It is to this topic that I devote the remainder of this section. An important quantity that I shall use to characterize a given model of distance fluctuations is the standard deviation of these fluctuations (which we expect to have zero mean). I shall place special emphasis on whether or not a given model predicts a standard deviation that grows with the time of observation. This type of standard deviation would reflect an element of decoherence (or "loss of information") intrinsic in space-time, in the sense that the information that an observer could store in a network of bodies by adjusting their distances to given values at a given initial time would degrade over time. ${ }^{3}$

\footnotetext{
${ }^{3}$ Other intuitive descriptions of the relation between certain foamy space-times and decoherence can be found in Ref. [26]. Depending on the reader's background it might also be useful to adopt the language of the "memory effect," as done, for example, in Ref. [27].
}

\section{B. Minimum-length noise and fuzziness with $T_{o b s}$-independent $\sigma$}

While the arguments on quantum-gravity-induced loss of quantum coherence appear to favor the possibility of decohering space-times (in the sense just discussed), there is an alternative viewpoint on quantum gravity, one in which the entire formalism of ordinary quantum mechanics is taken for granted, quantum coherence is automatically preserved and accordingly one is led to a nondecohering picture of spacetime (the standard deviation of distance fluctuations should be independent of the time of observation). The fact that these two formal intuitions (both presently unsupported by any data) lead to opposite expectations concerning the fundamental nature of space-time is not surprising. In fact, these two intuitions basically represent the two simplest approaches to the solution of the apparent incompatibility between gravitation and quantum mechanics. Decoherence naturally follows from assuming that the correct theory should preserve at least the most central elements of the general-relativistic description of gravitation, thereby forcing a modification of the laws of quantum mechanics. If instead one assumes that all the way down to the Planck length no change be necessary for the ordinary laws of quantum mechanics, some of the general-relativistic principles would have to be accordingly sacrificed $[1,13,14,28]$ eliminating the root of the arguments in support of decoherence.

In this subsection I shall review some of the arguments relevant for the type of space-time fuzziness which could be consistent with the exact preservation of all the axioms of quantum mechanics, while in the next subsection I shall consider the case of space-times with an intrinsic decoherence mechanism.

The starting point is the observation that in quantumgravity approaches based on ordinary quantum mechanics one naturally encounters a length scale $L_{\min }$, often identified with the string length $\left(L_{\text {string }} \sim 10^{-34} \mathrm{~m}\right)$ or the Planck length, which sets an absolute bound on the measurability of distances (a minimum uncertainty):

$$
\delta D \geqslant L_{\min } .
$$

This property emerges in approaches based on canonical quantization of Einstein's gravity when analyzing certain gedanken experiments (see, e.g., Refs. [29,30] and references therein). In critical superstring theories, theories whose mechanics is still governed by the laws of ordinary quantum mechanics but with one-dimensional (rather than point-like) fundamental objects, a relation of type (1) follows from the stringy modification of Heisenberg's uncertainty principle [31]

$$
\delta x \delta p \geqslant 1+L_{\text {string }}^{2} \delta p^{2} .
$$

In fact, whereas Heisenberg's uncertainty principle allows $\delta x=0$ (for $\delta p \rightarrow \infty$ ), for all choices of $\delta p$ the uncertainty relation (2) gives $\delta x \geqslant L_{\text {string. }}$. The relation (2) is suggested by certain analyses of string scattering [31], but it might have to be modified when taking into account the nonperturbative solitonic structures of superstrings known as Dirichlet branes 
[32]. In particular, evidence has been found [33] in support of the possibility that "Dirichlet particles" (Dirichlet 0 branes) could probe the structure of space-time down to scales shorter than the string length. In any case, all evidence available on critical superstring theory is consistent with a relation of type (1), although it is probably safe to say that some more work is still needed to firmly establish the stringtheory value of $L_{\min }$.

Having clarified that a relation of type (1) is a rather common prediction of quantum-gravity work assuming the validity of ordinary quantum mechanics, let me then consider how such a relation could affect the noise levels of an interferometer, i.e., let me consider the type of fuzziness which could be encoded in relation (1).

First let me observe that relation (1) does not necessarily encode any fuzziness; for example, relation (1) could simply emerge from a theory based on a lattice of points with spacing $L_{\min }$ and equipped with a measurement theory consistent with Eq. (1). The concept of distance in such a theory would not necessarily be affected by the type of stochastic processes that lead to noise in an interferometer.

However, it is also possible for relation (1) to encode the net effect of some underlying physical processes of the type one would qualify as quantum space-time fluctuations $[8,34-$ 37]. A very intuitive description of the way in which the dynamics of matter distributions would be affected by this type of fuzziness of space-time is obtained by noticing certain similarities [37] between a thermal environment and the environment of quantum space-time fluctuations consistent with Eq. (1). This (however preliminary) network of intuitions suggests that Eq. (1) could be the result of fuzziness for distances $D$ of the type associated with stochastic fluctuations with root-mean-square deviation $\sigma_{D}$ given by

$$
\sigma_{D} \sim L_{\min } .
$$

The fact that $\sigma_{D}$ is independent of the time of observation is consistent with the nature of the conceptual framework being considered in this subsection ("nondecohering space-time"). The associated displacement amplitude spectral density $S_{\min }(f)$ should roughly have a $1 / \sqrt{f}$ behavior

$$
S_{\min }(f) \sim \frac{L_{\min }}{\sqrt{f}} .
$$

This can be justified using the observation that for a frequency-band limited from below only by the time of observation $T_{o b s}$ the relation between $\sigma$ and $S(f)$ is given by [38]

$$
\sigma^{2}=\int_{1 / T_{o b s}}^{f_{\max }}[S(f)]^{2} d f
$$

Substituting the $S_{\min }(f)$ of Eq. (4) for the $S(f)$ of Eq. (5) one obtains a $\sigma$ that approximates the $\sigma_{D}$ of Eq. (3) up to small (logarithmic) $T_{o b s}$-dependent corrections. A more detailed description of the displacement amplitude spectral density associated with Eq. (3) can be found in Refs. [34,35]. For the objectives of the present article the rough estimate (4) is sufficient since, if indeed $L_{\min } \sim L_{p}$, from Eq. (4) one obtains $S_{\min }(f) \sim 10^{-35} \mathrm{~m} / \sqrt{f}$, which is still very far from the sensitivity of even the most advanced modern interferometers [and therefore we should not be concerned with small corrections to Eq. (4)].

\section{Distance fuzziness in space times with intrinsic decoherence mechanism}

The network of ideas relevant for the Wheeler-Hawking foamy or fuzzy picture of space-time and loss of quantum coherence has been discussed in numerous publications (a sample of recent ideas in this area can be found in Refs. $[39,36,40,41])$. However, while a substantial amount of work has been devoted to the "physics case" for quantum-gravity induced decoherence, enormous difficulties have been encountered in developing a satisfactory formalism for this type of quantum gravity. The primary obstruction for the search of the correct decoherence-encoding formalism is the fact that a new mechanics would be needed (ordinary quantum mechanics evolves pure states into pure states) and the indentification of such a new mechanics in the absence of any guidance from experiments is extremely hard. In particular, none of the quantum-gravity approaches emerged within this framework has been developed to the point of allowing a definite prediction for the quantum-gravity-induced amplitude spectral density of distance fluctuations. All one can really say at present is that in this alternative framework one can consider deviations from the $S(f) \sim f^{-1 / 2}$ behavior of nondecohering space-times, but the field is still quite open with respect to the nature and magnitude of these deviations. In this context a natural starting point for a phenomenological program investigating the possibility of quantum-gravityinduced distance fluctuations appears to be provided by the following class of candidate amplitude spectral densities: ${ }^{4}$

$$
S(f)=f^{-\beta}\left(\mathcal{L}_{\beta}\right)^{3 / 2-\beta} c^{\beta-1 / 2},
$$

where $c$ is the speed-of-light constant, the dimensionless parameter $\beta$ carries the information on the nature of the underlying stochastic processes and the dimensionful parameter $\mathcal{L}_{\beta}$ carries the information on the magnitude and rate of the fluctuations. ${ }^{5}$

In Eq. (6) the parameter $\beta$ could in principle take any value, and actually it is quite plausible that in reality the stochastic processes (if at all present) would have a more

\footnotetext{
${ }^{4}$ Of course, a parametrization such as the one in Eq. (6) could only be valid for frequencies $f$ large enough to be safely away from the singularity at $f=0$. One natural candidate for this required infrared cutoff appears to be provided by the inverse of the time or distance scale over which the classical geometry of the space-time region where the experiment is performed manifests significant curvature effects.

${ }^{5} \mathrm{I}$ am assigning an index $\beta$ to $\mathcal{L}_{\beta}$ just in order to facilitate a concise description of experimental bounds. For example, if data were to rule out the fluctuations scenario with, say, $\beta=0.6$ for all values of the effective length scale down to, say, $10^{-27} \mathrm{~m}$ one could simply write the formula $\mathcal{L}_{\beta=0.6}<10^{-27} \mathrm{~m}$.
} 
complex structure than the simple power law codified in Eq. (6). Still, at the present early stage in the investigation of short-distance properties of space-time Eq. (6) appears to be a good tool for the exploration of the possibility of "distance-fuzziness" effects induced (in the sense of Wheeler and Hawking) by quantum properties of space-time. In particular, it seems natural to devote special attention to values of $\beta$ in the range ${ }^{6} 1 / 2 \leqslant \beta \leqslant 1$; in fact, as explained above, $\beta=1 / 2$ is the type of behavior one would expect $[34,35]$ in fuzzy space-times without decoherence, while the case $\beta=1$ provides the simplest model of stochastic (quantum) fluctuations of distances, in which a distance is affected by completely random minute (possibly Planck-length size) fluctuations that can be modelled as stochastic processes of random-walk type. Values of $\beta$ somewhere in between the cases $\beta=1 / 2$ and $\beta=1$ could provide a rough model of space-times with decoherence effects somewhat milder than the $\beta=1$ random-walk case.

Readers unfamiliar with the subject can get an intuitive picture of the relation between the value of $\beta$ and the type of space-time decoherence here being considered by resorting again to Eq. (5). For example, as discussed in greater detail in Ref. [38], the case $\beta=1$ corresponds to $\sigma \sim \sqrt{T_{o b s}}$, as expected for random-walk stochastic processes, ${ }^{7}$ and therefore $\sigma$ does grow with the time of observation. Similar observations, but with weaker power-law dependence on $T_{\text {obs }}$, hold for values of $\beta$ in the range $1 / 2<\beta<1$. [As mentioned, in the limiting case $\beta=1 / 2$ the $T_{o b s}$ dependence turns from power-law to logarithmic, and this is of course the closest one can get to modeling space-times without decoherence within the parametrization set up in Eq. (6).]

In closing this subsection it seems worth adding a few comments on the stochastic processes here considered. For example, in most physical contexts a series of random steps does not lead to $\sqrt{T_{o b s}}$ dependence of $\sigma$ because often the nature of the problem is such that through the fluctuationdissipation theorem the original form of the $T_{o b s}$ dependence gets partly compensated. In other words, as exemplified by the phenomenon of Brownian motion, in most physical contexts involving one sort or another of random steps there is also a "restoring mechanism." Extrapolating this intuition to the point of imagining such a restoring mechanism associated with the intrinsic fluctuations of space-time itself is nontrivial. We are far enough from an understanding of the fundamental nature of space-time that such a restoring mechanism cannot be ruled out, but it also seems worth exploring the possibility that the type of underlying dynamics

\footnotetext{
${ }^{6}$ In addition, to the range $1 / 2 \leqslant \beta \leqslant 1$ it might be reasonable, since $\beta=0$ corresponds to the case of "white noise" (which is frequently encountered in various areas of physics), to also focus on values of $\beta$ between 0 and $1 / 2$. I postpone the discussion of these possibilities to future work.

${ }^{7}$ The interested reader can find several studies relevant to the general random-walk-noise relation between $\sigma \sim \sqrt{T_{o b s}}$ and $S(f)$ $\sim f^{-1}$. A good starting point is provided by Ref. [38].
}

of quantum space-time be such that the fluctuationdissipation theorem be satisfied without a restoring mechanism. This is an intuition which apparently is shared by other authors; in fact, the study reported in Ref. [42] (which followed by a few months Ref. [8], but clearly was the result of independent work) also models some implication of quantum space-time (the ones that affect time measurement) with stochastic processes whose underlying dynamics does not produce any dissipation and therefore the "fluctuation contribution" to the $T_{o b s}$ dependence remains unaffected, although the fluctuation-dissipation theorem is fully taken into account.

Assuming that the elementary stochastic process for space-time fluctuations can be modeled as some sort of random step, the parameter $\beta$ which I am using to characterize the $f$ dependence of the amplitude spectral density can be seen as parametrizing the strength of a possible restoring mechanism: without restoring mechanism a series of random steps with very short correlation would lead to $\beta=1$ while values of $\beta$ smaller than 1 could correspond to the presence of a restoring mechanism.

Another aspect of space-time fluctuations which might appear counterintuitive with respect to the experience of ordinary-physics stochastic processes emerges when applying the idea of space-time fluctuations to the mirrors of an interferometer. Since the mirrors of an interferometer are basically extremities of a pendulum, the reader might at first find counterintuitive that the $T_{o b s}$ dependence of $\sigma$ for extremely large $T_{o b s}$ would seem to give values of $\sigma$ too large to be consistent with the structure of a pendulum (even if suppressed by a very small prefactor, as it happens when the scale of the fluctuations is set by the Planck length, the $T_{o b s}$-dependent contribution to $\sigma$ can eventually become large for sufficiently large $T_{o b s}$ ). This is a misleading intuition which originates from the experience with ordinary (non-quantum-gravity) analyses of the pendulum. In fact, the dynamics of an ordinary pendulum has one extremity "fixed" to a very heavy and rigid body, while the other extremity is fixed to a much lighter body. The usual stochastic processes considered in the study of the pendulum affect the heavier body in a totally negligible way, while they have strong impact on the dynamics of the lighter body. Quantumgravity-induced distance fluctuations would plausibly affect a pendulum as stochastic processes which are of the same magnitude both for its heavier and its lighter extremity. (They are fluctuations of space-time itself rather than the result of some collisions with matter particles in a conventional classical space-time.) In particular in the directions orthogonal to the vertical axis the stochastic processes affect the position of the center of mass of the entire pendulum just as they would affect the position of the center of mass of any other body (the string that connects the two extremities of the pendulum would not affect the motion of its center of mass). With respect to the application of some of these considerations to modern gravity-wave interferometers it is also important to keep in mind that the measurement strategy of these interferometers requires that their test masses be freefalling. 


\section{SOME CANDIDATE FUZZINESS SCENARIOS}

As mentioned in the preceding section, while a substantial amount of work has been devoted to the "physics case" for quantum-gravity-induced decoherence, enormous difficulties have been encountered in developing a satisfactory formalism for this type of quantum gravity, mostly because one is seeking a new mechanics without any guidance from experiments. The general parametrization (6) reflects this lack of precise guidance from theory results. When a satisfactory workable formalism implementing the Wheeler-Hawking intuition on quantum-gravity-induced decoherence becomes available, we will be in a position to extract from it a specific form of the stochastic processes characterizing the associated foamy space-time, with a definite prediction for $S(f)$. In the meantime, besides seeking guidance from experiments, we can attempt to get some intuition for the short-distance properties of space-time by using heuristic arguments based on general expectations for the interplay between gravitation and quantum mechanics. In this section I use two types of such arguments. The first type is based on "in-principle studies" of the measurability of distances in quantum gravity, which are a recent development along the research line started by Salecker and Wigner ${ }^{8}$ in the 1950s [16], while the second type of arguments is based on a requirement of consistency with recent proposals $[4,19,20]$ of quantum-gravity

\footnotetext{
${ }^{8}$ The classic Salecker-Wigner work [16] is criticized in the paper [43] (i.e., long after the present article, had already been submitted for publication). As I explain in detail in Ref. [44], the analysis reported in Ref. [43] is incorrect. Whereas Salecker and Wigner sought an operative definition of distances suitable for the Planck regime, the analysis in Ref. [43] relies on several assumptions that appear to be natural in the context of most present-day experiments but are not even meaningful in the Planck regime. Moreover, contrary to the claim made in Ref. [43], the source of $\sqrt{T_{o b s}}$ uncertainty used in the Salecker-Wigner derivation cannot be truly eliminated; unsurprisingly, it can only be traded [44] for another comparable contribution to the total uncertainty in the measurement. In addition to this incorrect criticism of the limit derived by Salecker and Wigner, Ref. [43] also misrepresented the role of the SaleckerWigner limit in providing motivation for the interferometric studies here considered (and originally proposed in Ref. [8]): the reader could come out of reading Ref. [43] with the impression that such interferometry-based tests would only be sensitive to quantumgravity ideas motivated by the Salecker-Wigner limit. As emphasized in the present article motivation for this phenomenological program also comes from a long tradition of ideas (developing independently of the ideas related to the Salecker-Wigner limit) on foamy or fuzzy space-time, and from recent work on the possibility that quantum gravity might induce a deformation of the dispersion relation that characterizes the propagation of the massless particles used as space-time probes in the operative definition of distances. This is already quite clear at least to a portion of the community; for example, in recent work [45] on foamy space-times (without any reference to the Salecker-Wigner related literature) the type of modern-interferometer sensitivity exposed here and in Ref. [8] was used to constrain certain novel candidate quantum-gravity effects.
}

induced deformation of the dispersion relation that characterizes the propagation of the massless particles used as spacetime probes in the operative definition of distances.

\section{A. Random-walk noise motivated by the analysis of a Salecker-Wigner gedanken experiment}

In this subsection I consider some bounds on the measurability of distances which appear to emerge when taking into account the quantum properties of devices. It is well understood (see, e.g., Refs. [10,12,15,26,28,46,47]) that the combination of the gravitational properties and the quantum properties of devices can have an important role in the analysis of the operative definition of gravitational observables. Since the analyses $[29-31,33]$ that led to the proposal of Eq. (3) only treated the devices in a completely idealized manner (assuming that one could ignore any contribution to the uncertainty in the measurement of $D$ due to the gravitational and quantum properties of devices), it is not surprising that analyses that took into account the gravitational and quantum properties of devices found more significant limitations on the measurability of distances.

Actually, by ignoring the way in which the gravitational properties and the quantum properties of devices combine in measurements of geometry-related physical properties of a system one misses some of the fundamental elements of novelty we should expect for the interplay of gravitation and quantum mechanics; in fact, one would be missing an element of novelty which is deeply associated with the equivalence principle. In measurements of physical properties which are not geometry-related one can safely resort to an idealized description of devices. For example, in the famous Bohr-Rosenfeld analysis [48] of the measurability of the electromagnetic field it was shown that the accuracy allowed by the formalism of ordinary quantum mechanics could be achieved using idealized test particles with vanishing ratio between electric charge and inertial mass. Attempts to generalize the Bohr-Rosenfeld analysis to the study of gravitational fields (see, e.g., Ref. [15]) are of course confronted with the fact that the ratio between gravitational "charge", (mass) and inertial mass is fixed by the equivalence principle. While ideal devices with vanishing ratio between electric charge and inertial mass can be considered at least in principle, devices with vanishing ratio between gravitational mass and inertial mass are not admissible in any (however formal) limit of the laws of gravitation. This observation provides one of the strongest elements in support of the idea [12] that the mechanics on which quantum gravity is based must not be exactly the one of ordinary quantum mechanics, since it should accommodate a somewhat different relationship between "system" and "'measuring apparatus." [In particular, the new mechanics should not rely on the idealized measuring apparatus which plays such a central role in the mechanics laws of ordinary quantum mechanics, as illustrated by the "Copenhagen interpretation." ]

In trying to develop some intuition for the type of fuzziness that could affect the concept of distance in quantum gravity, it might be useful to consider the way in which the interplay between the gravitational and the quantum proper- 
ties of devices affects the measurability of distances. In Refs. $[10,12]$ I have argued ${ }^{9}$ that a natural starting point for this type of analysis is provided by the procedure for the measurement of distances which was discussed in influential work by Salecker and Wigner [16]. These authors "measured" (in the "gedanken" sense) the distance $D$ between two bodies by exchanging a light signal between them. The measurement procedure requires attaching ${ }^{10}$ a light-gun (i.e., a device capable of sending a light signal when triggered), a detector and a clock to one of the two bodies and attaching a mirror to the other body. By measuring the time $T_{o b s}$ (time of observation) needed by the light signal for a two-way journey between the bodies one also obtains a measurement of the distance $D$. For example, in Minkowski space and neglecting quantum effects one simply finds that $D$ $=c T_{o b s} / 2$. Within this setup it is easy to realize that the interplay between the gravitational and the quantum properties of devices leads to an irreducible contribution to the uncertainty $\delta D$. In order to see this it is sufficient to consider the contribution to $\delta D$ coming from the uncertainties that affect the motion of the center of mass of the system composed by the light-gun, the detector and the clock. Denoting with $x^{*}$ and $v^{*}$ the position and the velocity of the center of mass of this composite device relative to the position of the body to which it is attached, and assuming that the experimentalists prepare this device in a state characterized by uncertainties $\delta x^{*}$ and $\delta v^{*}$, one easily finds $[16,12]$

$$
\begin{aligned}
\delta D & \geqslant \delta x^{*}+T_{o b s} \delta v^{*} \geqslant \delta x^{*}+\left(\frac{1}{M_{b}}+\frac{1}{M_{d}}\right) \frac{\hbar T_{o b s}}{2 \delta x^{*}} \\
& \geqslant \sqrt{\frac{\hbar T_{o b s}}{2}\left(\frac{1}{M_{b}}+\frac{1}{M_{d}}\right)},
\end{aligned}
$$

where $M_{b}$ is the mass of the body, $M_{d}$ is the total mass of the device composed of the light-gun, the detector, and the clock, and the right-hand-side relation follows from observing that Heisenberg's uncertainty principle implies $\delta x^{*} \delta v^{*}$ $\geqslant\left(1 / M_{b}+1 / M_{d}\right) \hbar / 2$. [N.B.: the reduced mass $\left(1 / M_{b}\right.$ $\left.+1 / M_{d}\right)^{-1}$ is relevant for the relative motion.] Clearly, from Eq. (7) it follows that in order to eliminate the contribution to the uncertainty coming from the quantum properties of the devices it is necessary to take the formal "classical-device

\footnotetext{
${ }^{9}$ The Salecker-Wigner measurement procedure was also the starting point of the analyses reported in Refs. [26,49], which however approached the issue of measurability from a significantly different viewpoint (see comments in Sec. V).

${ }^{10}$ Of course, for consistency with causality, in such contexts one assumes devices to be "attached non-rigidly," and, in particular, the relative position and velocity of their centers of mass continue to satisfy the standard uncertainty relations of quantum mechanics.
}

limit," i.e., the limit ${ }^{11}$ of infinitely large $M_{d}$.

Up to this point I have not yet taken into account the gravitational properties of the devices and in fact the "classical-device limit" encountered above is fully consistent with the laws of ordinary quantum mechanics. From a physical or phenomenological and conceptual viewpoint it is well understood that the formalism of quantum mechanics is only appropriate for the description of the results of measurements performed by classical devices. It is therefore not surprising $^{12}$ that the classical-device (infinite-mass) limit turned out to be required in order to reproduce the prediction min $\delta D=0$ of ordinary quantum mechanics (which, as well known, allows $\delta A=0$ for any single observable $A$, since it only limits the combined measurability of pairs of conjugate observables).

If one also takes into account the gravitational properties of the devices, a conflict with ordinary quantum mechanics immediately arises because the classical-device (infinitemass) limit is in principle inadmissible for measurements concerning gravitational effects. As the devices get more and more massive they increasingly disturb the gravitational or geometrical observables, and well before reaching the infinite-mass limit the procedures for the measurement of gravitational observables cannot be meaningfully performed $[10,12,26]$. In the Salecker-Wigner measurement procedure the limit $M_{d} \rightarrow \infty$ is not admissible when gravitational interactions are taken into account. [At the very least the value of

\footnotetext{
${ }^{11} \mathrm{~A}$ body of finite mass can acquire a nearly-classical behavior when immerged in a suitable environment (environment-induced decoherence). However, one of the central hypothesis of the work of Salecker and Wigner and followers is that the quantum properties of devices should not be negligible in quantum gravity, and that in particular the in-principle operative definition of distances (which we expect to lie at the foundations of quantum gravity) should not rely on environment-induced decoherence. It appears worth exploring the implications of this hypothesis not only because quantum gravity could be a truly fundamental theory (rather than the effective large-distance description of a more fundamental theory) but also because the operative definition of distances in quantum gravity should be applicable all the way down to the Planck length. It is even plausible $[16,50]$ that quantum gravity should accommodate an operative definition of a material reference system composed of a network of free-falling particles with relative distances comparable to the Planck length. Within the framework of these intuitions it is indeed quite hard to imagine a decoherence-inducing environment suitable for the in-principle operative definition of distances in quantum gravity. As emphasized in Ref. [44], the analysis reported in Ref. [43] missed this important conceptual element of the Salecker-Wigner approach.

${ }^{12}$ Perhaps more troubling is the fact that $\min \delta D=0$ appears to require not only an infinitely large $M_{d}$ but also an infinitely large $M_{b}$. One feels somewhat uncomfortable treating the mass of the bodies whose distance is being measured as a parameter of the apparatus. This might be another pointer to the fact that quantum measurement of gravitational or geometric observables requires a novel conceptualization of quantum mechanics. I postpone the consideration of this point to future work.
} 
$M_{d}$ is limited by the requirement that the apparatus should not turn into a black hole, which would not allow the exchange of signals required by the measurement procedure.] These observations, which appear to render inaccessible the limit of vanishingly small right-hand side of Eq. (7), provide motivation for the possibility $[10,12]$ that in quantum gravity any measurement that monitors a distance $D$ for a time $T_{o b s}$ is affected by quantum fluctuations such that ${ }^{13}$

$$
\delta D \geqslant \sqrt{L_{Q G} c T_{o b s}},
$$

where $L_{Q G}$ could in principle be an independent fundamental length scale (a length scale characterizing the nature of the novel quantum-gravity relationship between system and apparatus), but one is tempted to consider the possibility that $L_{Q G}$ be simply related to the Planck length. Interestingly, according to Eq. (8) the Salecker-Wigner measurement of a distance $D$, which requires a time $2 D / c$, would be affected by an uncertainty of magnitude $\sqrt{L_{Q G} D}$.

Of course, the analyses reported above and in Refs. $[10,12]$ do not necessarily indicate that fuzziness of the type operatively defined in Sec. II should be responsible for the measurability bound (8). The intuitive or heuristic arguments I advocated can provide a (tentative) estimate of the measurability bound, but a full quantum-gravity theory woud be required in order to be able to determine which phenomena could be responsible for the bound. If one assumes that indeed fuzziness of the type operatively defined in Sec. II is responsible for the measurability bound (8) one is led to the possibility that a distance $D$ would be affected by fundamental stochastic fluctuations with the type of root-mean-square deviation $\sigma_{D}$ characteristic of "random walk noise" [38]:

$$
\sigma_{D} \sim \sqrt{L_{Q G} c T_{o b s}} .
$$

As discussed in Sec. II, the type of $T_{o b s}$ dependence of Eq. (9) corresponds to $\beta=1$, i.e.,

$$
S(f)=f^{-1} \sqrt{L_{Q G} c} .
$$

If indeed $L_{Q G} \sim L_{p}$, from Eq. (10) one obtains $S(f)$ $\sim f^{-1}\left(5 \times 10^{-14} \mathrm{~m} \sqrt{\mathrm{Hz}}\right)$. As I shall discuss in detail later, by the standards of modern interferometers this noise level is quite significant, and therefore, before discussing other estimates of distance fuzziness, let us see whether the naive guess $L_{Q G} \sim L_{p}$ can be justified within the argument used in

\footnotetext{
${ }^{13}$ Note that Eq. (8) sets a minimum uncertainty which takes only into account the quantum and gravitational properties of the measuring apparatus. Of course, an even tighter bound might emerge when taking into account also the quantum and gravitational properties of the system under observation. However, based on observations reported in Refs. [29,30] it appears likely that the contribution to the uncertainty coming from the system is of the type $\delta D$ $\geqslant L_{p}$, so that the total contribution (summing the system and the apparatus contributions) would be of the type $\delta D \geqslant L_{p}$ $+\sqrt{L_{Q G} c T_{o b s}}$ which in nearly all contexts of interest (satisfying $c T_{o b s} \gg L_{p}$ ) can be approximated by completely neglecting the $L_{p}$ correction originating from the properties of the system.
}

arriving at Eq. (8). Since Eq. (8) was motivated from Eq. (7), and in going from Eq. (7) to Eq. (8) the scale $L_{Q G}$ was introduced to parametrize the minimum allowed value of $1 / M_{b}+1 / M_{d}$, we could get some intuition for $L_{Q G}$ by trying to establish this minimum allowed value of $1 / M_{b}+1 / M_{d}$. As mentioned, a conservative (possibly very conservative) estimate of this minimum value can be obtained by enforcing that $M_{b}$ and $M_{d}$ be at least sufficiently small to avoid black hole formation. In leading order (e.g., assuming corresponding spherical symmetries) this amounts to the requirement that $M_{b}<\hbar S_{b} /\left(c L_{p}^{2}\right)$ and $M_{d}<\hbar S_{d} /\left(c L_{p}^{2}\right)$, where the lengths $S_{b}$ and $S_{d}$ characterize the sizes of the regions of space where the matter distributions associated with $M_{b}$ and $M_{d}$ are localized. This observation implies

$$
\frac{1}{M_{b}}+\frac{1}{M_{d}}>\frac{c L_{p}^{2}}{\hbar}\left(\frac{1}{S_{b}}+\frac{1}{S_{d}}\right)
$$

which in turn suggests [10] that $L_{Q G} \sim \min \left[L_{p}^{2}\left(1 / S_{b}+1 / S_{d}\right)\right]$ :

$$
\delta D \geqslant \min \sqrt{\left(\frac{1}{S_{b}}+\frac{1}{S_{d}}\right) \frac{L_{p}^{2} c T_{o b s}}{2}} .
$$

Of course, this estimate is very preliminary since a full quantum gravity would be needed here; in particular, the way in which black holes were handled in my argument might have missed important properties which could become clear only once we have the correct theory. However, it is nevertheless striking to observe that the naive guess $L_{Q G} \sim L_{p}$ appears extremely far from the intuition emerging from this estimate; in fact, $L_{Q G} \sim L_{p}$ would require that the maximum admissible value of $S_{d}$ (and $S_{b}$ ) be of order $L_{p}$. Since our analysis only holds for bodies and devices that can be treated as approximately rigid ${ }^{14}$ and any nonrigidity would introduce additional contributions to the uncertainties, it is reasonable to assume that $\max \left[S_{d}\right]$ be some small length (small enough that any nonrigidity would negligibly affect the measurement procedure), but the condition $\max \left[S_{d}\right] \sim L_{p}$ appears rather extreme. As I shall discuss in Sec. IV, already available experimental data rule out $L_{Q G} \sim L_{p}$ in Eq. (10), and therefore if the $f^{-1}$ dependence of Eq. (10) is verified in the physical world (which is of course only one of the possibilities) $\max \left[S_{d}\right]$ must be somewhat larger than $L_{p}$. As long as this type of analysis involves a $\max \left[S_{d}\right]$ which is independent of $\delta D$ one still finds $\sqrt{T_{o b s}}$ dependence of $\sigma_{D}$ [i.e., $f^{-1}$ dependence of $S(f)$ ]. If the correct quantum gravity is such that something like Eq. (12) holds but with $\max \left[S_{d}\right]$ that depends on $\delta D$ and/or $T_{o b s}$, one would have a different $T_{o b s}$ depen-

\footnotetext{
${ }^{14}$ The fact that I have included only one contribution from the quantum properties of the devices, the one associated with the quantum properties of the motion of the center of mass, implicitly relies on the assumption that the devices can be treated as approximately rigid. Any nonrigidity of the devices could introduce additional contributions to the uncertainty in the measurement of $D$.
} 
dence (and corresponding $f$ dependence), as I shall show in one example discussed in Sec. III D.

\section{B. Random-walk noise motivated by linear deformation of dispersion relation}

Another candidate quantum-gravity effect that provides some encouragement for the random-walk noise scenario has emerged in the context of studies of quantum-gravity induced $[4,19,20,51,52]$ deformation of the dispersion relation that characterizes the propagation of the massless particles used as space-time probes in the operative definition of distances.

Deformed dispersion relations are not uncommon in the quantum-gravity literature. For example, they emerge naturally in quantum-gravity scenarios requiring a modification of Lorentz symmetry. Modifications of Lorentz symmetry could result from space-time discreteness, a possibility extensively investigated in the quantum-gravity literature (see, e.g., Refs. [53]), and it would also naturally result from an "active" quantum-gravity vacuum of the type advocated by Wheeler and Hawking (such a vacuum might physically label the space-time points).

While most quantum-gravity approaches will lead to deformed dispersion relations, the specific structure of the deformation can differ significantly from model to model. Assuming that the deformation admits a series expansion at small energies $E$, and parametrizing the deformation in terms of an energy ${ }^{15}$ scale $E_{Q G}$ (a scale, often identified with the Planck energy $E_{p} \sim 10^{19} \mathrm{GeV}$, characterizing the onset of strong quantum-gravity dispersion effects), one would expect to be able to approximate the deformed dispersion relation at low energies according to

$$
c^{2} \mathbf{p}^{2} \simeq E^{2}\left[1+\xi\left(\frac{E}{E_{Q G}}\right)^{\alpha}\right]
$$

where the power $\alpha$ and the sign ambiguity $\xi= \pm 1$ would be fixed in a given dynamical framework. For example, in some of the approaches based on dimensionful " $\kappa$ " quantum deformations of Poincaré symmetries [51,52] one finds evidence of a dispersion relation for massless particles $c^{2} \mathbf{p}^{2}$ $=E_{Q G}^{2}\left[1-e^{E / E_{Q G}}\right]^{2}$, and therefore $\xi=\alpha=1$.

Scenarios (13) with $\alpha=1$ are in a sense consistent with random-walk noise. In fact, an experiment involving as a device (as a probe) a massless particle satisfying the dispersion relation (13) with $\alpha=1$ would be naturally affected by a device-induced uncertainty that grows with $\sqrt{T_{o b s}}$. This is for example true in quantum-gravity scenarios in which the Hamiltonian equation of motion $\dot{x}_{i}=\partial H / \partial p_{i}$ is still valid (at least approximately), where the deformed dispersion relation (13) leads to energy-dependent velocities for massless particles

\footnotetext{
${ }^{15}$ I parametrize deformations of dispersion relations in terms of an energy scale $E_{Q G}$, while I parametrize the proposals for measurability bounds with a length scale $L_{Q G}$.
}

$$
v \simeq c\left[1-\left(\frac{1+\alpha}{2}\right) \xi\left(\frac{E}{E_{Q G}}\right)^{\alpha}\right]
$$

and consequently the uncertainty in the position of the massless probe when a time $T_{o b s}$ has lapsed since the observer (experimentalist) set off the measurement procedure is given by

$$
\delta x \simeq c \delta t+\delta v T_{o b s} \simeq c \delta t+\frac{1+\alpha}{2} \alpha \frac{E^{\alpha-1} \delta E}{E_{Q G}^{\alpha}} c T_{o b s},
$$

where $\delta t$ is the uncertainty in the time of emission of the probe, $\delta v$ is the uncertainty in the velocity of the probe, $\delta E$ is the uncertainty in the energy of the probe, and I used the relation between $\delta v$ and $\delta E$ that follows from Eq. (14). Since the uncertainty in the time of emission of a particle and the uncertainty in its energy are related ${ }^{16}$ by $\delta t \delta E \geqslant \hbar$, Eq. (15) can be turned into an absolute bound on the uncertainty in the position of the massless probe when a time $T_{o b s}$ has lapsed since the observer set off the measurement procedure:

$$
\begin{aligned}
\delta x & \geqslant c \frac{\hbar}{\delta E}+\frac{1+\alpha}{2} \alpha \frac{E^{\alpha-1} \delta E}{E_{Q G}^{\alpha}} T_{o b s} \\
& \geqslant \sqrt{\left(\frac{\alpha+\alpha^{2}}{2}\right)\left(\frac{E}{E_{Q G}}\right)^{\alpha-1} \frac{c^{2} \hbar T_{o b s}}{E_{Q G}}},
\end{aligned}
$$

where I also used the fact that in principle the observer can prepare the probe in a state with desired $\delta E$, so it is legitimate to minimize the uncertainty with respect to the free choice of $\delta E$.

For $\alpha=1$ the $E$ dependence on the right-hand side of Eq. (16) disappears and one is led again to a $\delta x$ of the type const $\times \sqrt{T_{\text {obs }}}$ :

$$
\delta x \geqslant \sqrt{\frac{c^{2} \hbar T_{o b s}}{E_{Q G}}} .
$$

When massless probes are used in the measurement of a distance $D$ the uncertainty (17) in the position of the probe translates directly into an uncertainty on $D$ :

$$
\delta D \geqslant \sqrt{\frac{c^{2} \hbar T_{o b s}}{E_{Q G}}} .
$$

\footnotetext{
${ }^{16}$ It is well understood that the $\delta t \delta E \geqslant \hbar$ relation is valid only in a weaker sense than, say, Heisenberg's uncertainty principle $\delta x \delta p$ $\geqslant \hbar$. This has its roots in the fact that the time appearing in quantum-mechanics equations is just a parameter (not an operator), and in general there is no self-adjoint operator canonically conjugate to the total energy, if the energy spectrum is bounded from below [42,54]. However, $\delta t \delta E \geqslant \hbar$ does relate $\delta t$ intended as uncertainty in the time of emission of a particle and $\delta E$ intended as uncertainty in the energy of that same particle.
} 
This was already observed in Refs. [11,19,52] which considered the implications of deformed dispersion relations (13) with $\alpha=1$ for the operative definition of distances.

If we assume again that such measurability bounds emerge in a full quantum gravity as a result of corresponding quantum fluctuations (fuzziness), we are led once again to random-walk noise:

$$
\sigma_{D} \sim \sqrt{\frac{c^{2} \hbar T_{o b s}}{E_{Q G}}} .
$$

\section{Noise motivated by quadratic deformation of dispersion relation}

In the preceding Sec. IIIC I observed that quantumgravity deformed dispersion relations (13) with $\alpha=1$ can also motivate random-walk noise $\sigma_{D} \sim$ const $\times \sqrt{T_{o b s}}$. If we use the same line of reasoning that connects a measurability bound to a scenario for fuzziness when $\alpha \neq 1$ we find $\sigma_{D}$ $\sim \mathcal{G}\left(E / E_{Q G}\right) \sqrt{T_{o b s}}$, where $\mathcal{G}\left(E / E_{Q G}\right)$ is a ( $\alpha$-dependent) function of $E / E_{Q G}$. However, in these cases with $\alpha \neq 1$ clearly the connection between measurability bound and fuzzy-distance scenario cannot be too direct; in fact, this connection appears to require a counterpart in the fuzzy-distance scenario for the dependence of the measurability bound on the energy $E$ of the probe. One possibility is that if $\alpha \neq 1$ the interferometer noise levels induced by space-time fuzziness might be of the type [see Eq. (16)]

$$
\sigma_{D} \sim \sqrt{\left(\frac{\alpha+\alpha^{2}}{2}\right)\left(\frac{E^{*}}{E_{Q G}}\right)^{\alpha-1} \frac{c^{2} \hbar T_{o b s}}{E_{Q G}}},
$$

where $E^{*}$ is some energy scale characterizing the physical context under consideration. [For example, at the intuitive level one might conjecture that $E^{*}$ could characterize some sort of energy density associated with quantum fluctuations of space-time or an energy scale associated with the masses of the devices used in the measurement process.]

Since $\alpha \geqslant 1$ in all quantum-gravity approaches believed to support deformed dispersion relations, and since it is quite plausible that $E_{Q G}$ would be rather close to $10^{19} \mathrm{GeV}$, it appears likely that the factor $\left(E^{*} / E_{Q G}\right)^{\alpha-1}$ would suppress the random-walk noise effect.

\section{Noise with $f^{-5 / 6}$ amplitude spectral density}

In this subsection I discuss an argument that suggests the possibility: $\delta D \sim T_{o b s}^{1 / 3}$. This is formally the same type of relation obtained from a different viewpoint (see comments in Sec. V) in Refs. [26,49]. Importantly, the $f^{-5 / 6}$ amplitude spectral density that was derived for this scenario in Ref. [8] turns out to compare very interestingly with experimental data, in the sense that, as clarified in the next section, presently available data fall just short of probing this possibility while the experiments that will soon start operating can provide rather sensitive tests.

The way in which I shall here obtain the $T_{o b s}^{1 / 3}$ behavior is based on the observation I made in Sec. III A that a relevant measurability bound could be derived by simply insisting that the devices do not turn into black holes. That observation allowed to derive Eq. (12), which expresses the minimum uncertainty $\delta D$ on the measurement of a distance $D$ (i.e., the measurability bound for $D$ ) as proportional to $\sqrt{T_{o b s}}$ and $\sqrt{\left(1 / S_{b}+1 / S_{d}\right)}$. Within that derivation the minimum uncertainty is therefore obtained in correspondence of the minimum value of $1 / S_{b}+1 / S_{d}$ consistent with the structure of the measurement procedure. I was led to consider how large $S_{d}$ could be while still allowing to disregard any nonrigidity in the quantum motion of the device (which would otherwise lead to additional contributions to the uncertainties). I found some motivation for the random-walk noise scenario by simply assuming that $\max \left[S_{d}\right]$ be independent of $T_{o b s}$ and independent of the accuracy $\delta D$ that the observer would wish to achieve. However, as already argued earlier in this article, the same physical intuition that motivates some of the fuzzy space-time scenarios here considered also suggests that quantum gravity might require a novel measurement theory, possibly involving a new type of relationship between system and measuring apparatus. Based on this intuition, it seems reasonable to contemplate the possibility that $\max \left[S_{d}\right]$ might actually depend on $\delta D$.

It is such a scenario that I want to consider in this subsection. In particular I want to consider the case $\max \left[S_{d}\right]$ $\sim \delta D$, which, besides being simple, has the plausible property that it allows only small devices if the uncertainty to be achieved is small, while it would allow correspondingly larger devices if the observer was content with a larger uncertainty. This is also consistent with the idea that elements of nonrigidity in the quantum motion of extended devices might be negligible if anyway the measurement is not aiming for great accuracy, while they might even lead to the most significant contributions to the uncertainty if all other sources of uncertainty are very small. It also seems plausible that "large" devices would not be suitable for very accurate space-time measurements while they might be admissible if space-time is being probed rather softly.

In this scenario with $\max \left[S_{d}\right] \sim \delta D$, Eq. (12) takes the form

$$
\delta D \geqslant \sqrt{\left(\frac{1}{S_{b}}+\frac{1}{S_{d}}\right) \frac{L_{p}^{2} c T_{o b s}}{2}} \geqslant \sqrt{\frac{L_{p}^{2} c T_{o b s}}{2 \delta D}},
$$

which actually gives

$$
\delta D \geqslant\left(\frac{1}{2} L_{p}^{2} c T_{o b s}\right)^{1 / 3} .
$$

As already done with the other measurability bounds discussed in this article, I shall take Eq. (22) as motivation for the investigation of the corresponding fuzziness scenarios characterized by

$$
\sigma_{D} \sim\left(\widetilde{L}_{Q G}^{2} c T_{o b s}\right)^{1 / 3} .
$$

Notice that in this equation I replaced $L_{p}$ with a generic length scale $\widetilde{L}_{Q G}$, since it is possible that the heuristic argument leading to Eq. (23) might have captured the qualitative structure of the phenomenon while providing an incorrect 
estimate of the relevant length scale. As discussed later in this article significant bounds on this length scale can be set by experimental data, so we can take a phenomenological attitude toward $\widetilde{L}_{Q G}$.

As one can verify for example using Eq. (5), the $T_{o b s}^{1 / 3}$ dependence of $\sigma_{D}$ is associated [8] with displacement amplitude spectral density with $f^{-5 / 6}$ behavior:

$$
\mathcal{S}(f)=f^{-5 / 6}\left(\widetilde{L}_{Q G}^{2} c\right)^{1 / 3} .
$$

For $\mathcal{L}_{\beta=5 / 6} \equiv \widetilde{L}_{Q G} \sim 10^{-35} \mathrm{~m}$ (note that $\widetilde{L}_{Q G}$ corresponds to $\mathcal{L}_{\beta=5 / 6}$ in the notation set up in Sec. II) this equation would predict $\mathcal{S}(f)=f^{-5 / 6}\left(3 \times 10^{-21} \mathrm{mHz}^{1 / 3}\right)$.

\section{COMPARISON WITH GRAVITY-WAVE INTERFEROMETER DATA}

In this section I discuss the potential of modern interferometers for the investigation of the two-parameter space of Eq. (6). In giving intuition for the significance of the bounds that can be obtained using these interferometers I shall often refer to three representative cases: the case $\beta=1 / 2$, corresponding to the hypothesis that space-time would not host any decoherence in the sense discussed in Sec. II, the case $\beta=1$, corresponding to the simple random-walk scenario for space-time fluctuations for which we found additional (however heuristic and indirect) motivation in the preceding section, and the case $\beta=5 / 6$, representative of all the scenarios with $1 / 2<\beta<1$ and being singled out by the additional supporting argument discussed in the preceding section. I shall freely switch back and forth between the notation adopted in Sec. II and the notation adopted in Sec. III: $\mathcal{L}_{\beta=1 / 2} \equiv L_{\text {min }}$, $\mathcal{L}_{\beta=1} \equiv L_{Q G}, \mathcal{L}_{\beta=5 / 6} \equiv \widetilde{L}_{Q G}$.

The discussion of the fuzziness scenarios considered in the preceding sections was consistent with the assumption that the length scale $\mathcal{L}_{\beta}$ characterizing fuzziness would be a general fundamental property of quantum gravity, independent of the peculiarities of the specific experimental context. However, as illustrated by the discussion in Secs. III A and III C, it is also plausible that $\mathcal{L}_{\beta}$ would not be a universal length scale, i.e. it might depend on some specific properties of the experimental context. The possibility that the "magnitude" of space-time fuzziness might depend on the specific context and experimental setup is also consistent with the arguments which support the possibility of a novel quantumgravity relationship between system and measuring apparatus. If the length scale characterizing fuzziness depended on this relationship it might take different values in different experimental setups.

Postponing to future phenomenological studies this possibility of context-dependent value of the scale characterizing the magnitude of the distance fluctuations, I shall here discuss the bounds set on $\mathcal{L}_{\beta}$ by data collected at the Caltech 40-meter interferometer. Before I do that, let me observe that, while conceptually they represent drastic departures from conventional physics, phenomenologically distance fluctuations of the type described by Eq. (6) could encode only minute effects. For example, for $\mathcal{L}_{\beta} \sim L_{p}$ the corre- sponding fluctuations would lead, for all values of $\beta$, to a standard deviation not greater than $10^{-5} \mathrm{~m}$ on a time of observation as large as $10^{10}$ years (and the size of the whole observable universe is about $10^{10}$ light years). However, the precision [21] of modern interferometers is such that they can provide significant information at least on the scenarios with values of $\beta$ toward the high end of the interesting interval $1 / 2 \leqslant \beta \leqslant 1$. In fact, as already mentioned in Sec. II, the operation of interferometers is based on the detection of minute changes in the positions of some test masses (relative to the position of a beam splitter). If these positions were affected by quantum fluctuations of the type discussed above the operation of interferometers would effectively involve an additional source of noise due to quantum-gravity. This observation allows to set interesting bounds already using existing noise-level data obtained at the Caltech 40-meter interferometer, which has achieved [22] displacement noise levels with amplitude spectral density lower than $10^{-18} \mathrm{~m} / \sqrt{\mathrm{Hz}}$ for frequencies between 200 and $2000 \mathrm{~Hz}$. As seen by straightforward comparison with Eqs. (6),(10) these sensitivity levels clearly rule out all values of $L_{Q G}$ (i.e., $\mathcal{L}_{\beta=1}$ ) down to the Planck length. Actually, even values of $L_{Q G}$ significantly smaller than the Planck length are inconsistent with the data reported in Ref. [22]; in particular, by confronting Eqs. (6),(10) with the observed noise level of $3 \times 10^{-19} \mathrm{~m} / \sqrt{\mathrm{Hz}}$ near $450 \mathrm{~Hz}$, which is the best achieved at the Caltech 40-meter interferometer, one obtains the bound $L_{Q G} \leqslant 10^{-40} \mathrm{~m}$.

In order to get some intuition for the significance of this bound let me observe that a value of $L_{Q G} \equiv L_{\beta=1}$ $\sim 10^{-35} \mathrm{~m}$ would correspond to fluctuations in the 40-meter arms of the Caltech interferometer that are of Planck-length magnitude and occur at a rate of one per each Planck-time interval. The data obtained at the Caltech 40-meter interferometer therefore rule out this simple random-walk model in spite of the minuteness (Planck-length) of the fluctuations involved. This is perhaps the most intuitive way to characterize the sensitivity of modern interferometers to distance fuzziness. Not long ago it might have seemed impossible to test a scenario involving fluctuations of magnitude $L_{p}$.

While the bounds on the $\beta=1$ scenario are impressive, for the case $\beta=1 / 2$, the case providing an effective model for space-times without intrinsic decoherence, the sensitivity levels achieved at the Caltech 40-meter interferometer only imply $\mathcal{L}_{\beta=1 / 2}<10^{-17} \mathrm{~m}$, which is still very comfortably consistent with the natural expectation $[34,35]$ that within that framework one would have $\mathcal{L}_{\beta=1 / 2} \sim L_{p} \sim 10^{-35} \mathrm{~m}$.

The sensitivity levels achieved at the Caltech 40-meter interferometer do allow to set an interesting bound on the scenario $\beta=5 / 6$. By observing that for $\beta=5 / 6$ one would predict quantum-gravity-induced noise levels for interferometers of order $\widetilde{L}_{Q G}^{2 / 3}\left(10 \mathrm{~m}^{1 / 3} / \sqrt{\mathrm{Hz}}\right)$ at frequencies of a few hundred $\mathrm{Hz}$, one obtains from the data reported in Ref. [22] that $\widetilde{L}_{Q G} \leqslant 10^{-29} \mathrm{~m}$. This bound is remarkably stringent in absolute terms, but is still quite far from the range of values one ordinarily considers as likely candidates for length scales appearing in quantum gravity. A more significant bound on $\widetilde{L}_{Q G}$ should be obtained by the Laser Interferometric Gravi- 
tational Wave Observatory (LIGO) or VIRGO generation of gravity-wave interferometers. For example, it is plausible [23] that the "advanced phase" of LIGO achieve displacement noise levels with amplitude spectral density of less than $10^{-20} \mathrm{~m} / \sqrt{\mathrm{Hz}}$ near $100 \mathrm{~Hz}$ and this would probe values of $\widetilde{L}_{Q G}$ as small as $10^{-34} \mathrm{~m}$.

Having discussed the three chosen representative cases, $\beta=1 / 2, \beta=1$ and $\beta=5 / 6$, let me now provide a general formula encoding the level of sensitivity achieved at the Caltech 40-meter interferometer as a function $\beta$. The data obtained at the Caltech 40-meter interferometer, which in particular achieved [22] displacement noise levels with amplitude spectral density of about $3 \times 10^{-19} \mathrm{~m} / \sqrt{\mathrm{Hz}}$ in the neighborhood of $450 \mathrm{~Hz}$, allow us to set the bound $[8,9]$

$$
\left[\mathcal{L}_{\beta}\right]_{\text {Caltech }}<\left[\frac{3 \times 10^{-19} \mathrm{~m}}{\sqrt{\mathrm{Hz}}}(450 \mathrm{~Hz})^{\beta} c^{(1-2 \beta) / 2}\right]^{2 /(3-2 \beta)} .
$$

Note that the bound encoded in Eq. (25) becomes less stringent as the value of $\beta$ decreases.

For all values of $\beta$ we can expect to establish more significant bounds using the LIGO-VIRGO generation $[23,24]$ of interferometers. Looking beyond the LIGO-VIRGO generation of gravity-wave interferometers, one can envisage still quite sizeable margins for improvement by optimizing the performance of the interferometers at low frequencies. It appears natural to perform such studies in the quiet environment of space, perhaps through future refinements of Laser Interferometer Space Anenna- (LISA)-type setups [25].

Interferometers are our best long-term hope for the development of this phenomenology (including the exploration of possible context-dependent $L_{\beta}$ ), and that is why the analysis in this article focuses on interferometers. However, it should be noted that among detectors already in operation the best bound on $L_{\beta}$ (if taken as universal) comes from resonant-bar detectors such as NAUTILUS [55], which achieved displacement sensitivity of about $10^{-21} \mathrm{~m} / \sqrt{\mathrm{Hz}}$ near $924 \mathrm{~Hz}$. Correspondingly, one obtains the bound

$$
\left[\mathcal{L}_{\beta}\right]_{\text {bars }}<\left[\frac{10^{-21} \mathrm{~m}}{\sqrt{\mathrm{Hz}}}(924 \mathrm{~Hz})^{\beta} c^{(1-2 \beta) / 2}\right]^{2 /(3-2 \beta)} .
$$

The indication of the low-frequency range as significant for quantum-gravity tests at interferometers is perhaps the most intruiging point made in this article. The arguments advocated in the previous Secs. II and III were all rather heuristic and it would not be surprising if some of the details of the estimates turned out to be completely off the mark, but the fact that all of those arguments pointed us toward the low-frequency region might nevertheless be indicative.

\section{RELATIONS WITH OTHER QUANTUM GRAVITY APPROACHES}

The general strategy for the search of quantum gravity which has led to the arguments reviewed and/or presented in the previous sections is evidently quite different from the strategy adopted in other approaches to the unification of gravity and quantum mechanics. [I shall discuss these differences in greater detail in Sec. VII.] However, it is becoming increasingly clear (especially in discussions and research papers that were motivated by Refs. $[4,8]$ ) that in spite of these differences some common elements of intuition concerning the interplay of gravitation and quantum mechanics are emerging. In this section I want to emphasize these relationships with some quantum-gravity approaches and at the same time I want to clarify the differences with respect to other quantum-gravity approaches.

\section{A. Canonical quantum gravity}

One of the most popular approaches to the unification of gravity and quantum mechanics (whose popularity might have been the reason for the diffusion of the possibly misleading name "quantum gravity") is the one in which the ordinary canonical formalism of quantum mechanics is applied to (some formulation of) Einstein's general-relativistic theory of gravitation.

While I must emphasize again [12] that some of the observations reviewed and/or reported in the previous sections strongly suggest that quantum gravity should require a new mechanics, not exactly given by ordinary quantum mechanics, it is nonetheless encouraging ${ }^{17}$ that some of the phenomena considered in the previous sections have also emerged in studies of canonical quantum gravity.

The most direct connection was found in the study reported in Ref. [20], which was motivated by Ref. [4]. In fact, Ref. [20] shows that the popular canonical or loop quantum gravity [56] admits (under certain conditions, which in particular involve some parity breaking) the phenomenon of deformed dispersion relations with the deformation going linearly with the Planck length.

Concerning the bounds on the measurability of distances it is probably fair to say that the situation in canonical or loop quantum gravity is not yet clear because the present formulations do not appear to lead to a compelling candidate "length operator." This author would like to interpret the problems associated with the length operator as an indication that perhaps something unexpected might actually emerge in canonical or loop quantum gravity as a length operator, possibly something with properties fitting the intuition emerging from the analyses in Secs. II C, III A, and III D. Actually, the

\footnotetext{
${ }^{17} \mathrm{I}$ am here taking a viewpoint that might be summarized rephrasing a comment by De Witt in Ref. [28]. While some of the arguments reviewed here appear to indicate that ordinary quantum mechanics cannot suffice for quantum gravity, it is still plausible that the language of ordinary quantum mechanics might be a useful tool for the description of its own demise. This would be analogous to something we have learned in the study of special relativity: one could [28] insist on describing the observed Lorentz-Fitzgerald contraction as the result of relativistic modifications in the force law between atoms, but in order to capture the true essence of the new regime it is necessary to embrace the new conceptual framework of special relativity.
} 
random-walk space-time fuzziness model discussed in Sec. II C might have a (still somewhat vague, but intriguing) connection with "quantum mechanics applied to gravitation" at least to the level seen by comparison with the scenario discussed in Ref. [57], which was motivated by the intuition that is emerging from investigations of the canonical or loop quantum gravity. The "moves" of Ref. [57] share many of the properties of the "random steps" of the random-walk models here considered. Unfortunately, in both approaches one is still searching for a more complete description of the dynamics.

\section{B. Noncommutative geometry and deformed symmetries}

Although this was not emphasized in the present article, some of the quantum-gravity intuition emerging from the observations in the previous sections fits rather naturally within certain approaches based on noncommutative geometry and deformed symmetries. In particular, there is growing evidence $[11,52]$ that theories living in the noncommutative Minkowski space proposed in Refs. [58,51], which involves a dimensionful (possibly Planck length related) deformation parameter, would host both Planck-length-linear deformations of dispersion relations and $T_{o b s}$-dependent bounds on the measurability of distances.

In general, the possibility of dimensionful deformations of symmetries [51,59] might be quite natural [12] if indeed the relation between system and measuring apparatus is modified at the quantum-gravity level. For example, the symmetries we observe in ordinary quantum-mechanics experiments at low energies might be the ones valid in the limit in which the interaction between system and measuring apparatus can be neglected. The dimensionful parameter characterizing the deformation of symmetries could mark a clear separation between (high-energy) processes, in which the violations of ordinary symmetries are large, and (low-energy) processes, in which ordinary symmetries hold to a very good approximation.

On the subject of quantum deformations of space-time symmetries interesting work has also been devoted (see, e.g., Refs. $[60,61])$ to frameworks that would host a bound on the measurability of distances of type (1).

\section{Critical and noncritical string theories}

Unfortunately, in the popular quantum-gravity approach based on critical superstring theory ${ }^{18}$ not many results have been derived concerning directly the quantum properties of space-time. Perhaps the most noticeable such results are the ones on limitations on the measurability of distances emerged in the scattering analyses reported in Refs. [31,33], which I already mentioned in Sec. II B, since they provide support for the hypothesis that also critical superstring theory might host a bound on the measurability of distances of type (1).

\footnotetext{
${ }^{18}$ As already mentioned the mechanics of string theory is just an ordinary quantum mechanics. The novelty of the approach comes from the fact that the fundamental dynamical entities are extended objects rather than point particles.
}

A rather different picture is emerging (through the difficult technical aspects of this rich formalism) in Liouville (noncritical) string theory [18], whose development was partly motivated by intuition concerning the "quantumgravity vacuum" that is rather close to the one traditionally associated with the works of Wheeler [13] and Hawking [14]. Evidence has been found [19] in Liouville String Theory supporting the validity of deformed dispersion relations, with the deformation going linearly with the Planck or string length. In the sense clarified in Sec. III B this approach might also host a bound on the measurability of distances which grows with $\sqrt{T_{\text {obs }}}$.

\section{Other types of measurement analyses}

In light of the scarcity of opportunities to get any experimental input in the search for quantum gravity, it is not surprising that many authors have been seeking some intuition by formal analyses of the ways in which the interplay between gravitation and quantum mechanics could affect measurement procedures. A large portion of these analyses produced a " $\min [\delta D]$ " with $D$ denoting a distance; however, the same type of notation was used for structures defined in significantly different manner. Also different meanings have been given by different authors to the statement "absolute bound on the measurability of an observable." Quite important for the topics here discussed are the differences (which might not be totally transparent as a result of this unfortunate choice of overlapping notations) between the approach advocated in the present article (and in Refs. [8,10,12]) and the approaches advocated in Refs. [16,26,46,49]. In the present article " $\min [\delta D]$ " denotes an absolute limitation on the measurability of a distance $D$. The studies $[16,46,49]$ analyzed the interplay of gravitation and quantum mechanics in defining a net of timelike geodesics, and in those studies " $\min [\delta D]$ " characterizes the maximum "tightness" achievable for the net of timelike geodesics. Moreover, in Refs. $[16,26,46,49]$ it was required that the measurement procedure should not affect or modify the geometric observable being measured, and "absolute bounds on the measurability" were obtained in this specific sense. Instead, here and in Refs. $[10,12]$ I allowed the possibility for the observable which is being measured to depend also on the devices (the underlying view is that observables in quantum gravity would always be, in a sense, shared properties of "system" and "apparatus"), and I only required that the nature of the devices be consistent with the various stages of the measurement procedure (e.g., a black-hole device would not allow some of the required exchanges of signal). My measurability bounds are therefore to be intended from this more fundamental perspective, and this appears to be important for the possibility that these measurability bounds be associated to a fundamental quantum-gravity mechanism for "fuzziness" (quantum fluctuations of space-time). The analyses reported in Refs. [16,26,46,49] did not include any reference to fuzzy space-times of the type operatively defined in Sec. II.

The more fundamental nature of the bounds I obtained is also crucial for the arguments suggesting $[10,12]$ that quantum gravity might require a new mechanics, not exactly 
given by ordinary quantum mechanics. The analyses reported in Refs. $[16,26,46,49]$ did not include any reference to this possibility.

I also notice that the relation (considered here and in Ref. [8]) between measurability bounds and noise levels in interferometers [e.g., the ones characterized by $S(f) \sim f^{-1}$ or $S(f) \sim f^{-5 / 6}$ ] is based on the dependence of the measurability bounds on the time of observation $T_{o b s}$. In fact, this $T_{o b s}$ dependence [and the $f$ dependence of the amplitude spectral density $S(f)$ ] has been here emphasized, while in Refs. $[26,46,49]$ the emphasis was placed on observed lengths rather than on the time needed to observe them [and accordingly in Refs. $[26,46,49]$ neither the amplitude spectral density $S(f)$ nor any other similar structure was considered].

Having clarified that there is a "double difference"' (different "min" and different " $\delta D$ ') ) between the meaning of $\min [\delta D]$ adopted in the present article and the meaning of $\min [\delta D]$ adopted in Refs. [16,26,46,49], it is however important to notice that the studies reported in Refs. [26,46,49] were among the first studies which showed how in some aspects of measurement analysis the Planck length might appear together with other length scales in the problem. For example, a quantum-gravity effect naturally involving something of length-squared dimensions might not necessarily go like $L_{p}^{2}$, in some cases it could go like $\Lambda L_{p}$, with $\Lambda$ some other length scale in the problem. Some of my arguments are examples of this possibility; in particular, I find in some cases relations of the type [see, e.g., Eq. (7)]

$$
\delta D \geqslant \delta x^{*}+\frac{A}{\delta x^{*}} \geqslant \sqrt{A},
$$

where $A$, which has length-squared dimensions, turns out to be given by the product of the $L_{p}$-like small fundamental length $L_{Q G}$ and the typically larger length scale $c T_{o b s}$.

Interestingly, the analysis of the interplay of gravitation and quantum mechanics in defining a net of timelike geodesics reported in Ref. [46] concluded that the maximum "tightness" achievable for the geodesics would be characterized by $\sqrt{L_{p}^{2} R^{-1} s}$, where $R$ is the radius of the (spherically symmetric) clocks whose world lines define the network of geodesics, and $s$ is the characteristic distance scale over which one is intending to define such a network. The $\sqrt{L_{p}^{2} R^{-1} s}$ maximum tightness discussed in Ref. [46] is formally analogous to my Eq. (12), but, as clarified above, this "maximum tightness" was defined in a way that is very ("doubly") different from my " $\min [\delta D]$," and therefore the two proposals have completely different physical implications. Actually, in Ref. [46] it was also stated that for a single geodesic distance (which might be closer to the type of distance measurability analysis reported here and in Refs. $[10,12])$ one could achieve accuracy significantly better than the formula $\sqrt{L_{p}^{2} R^{-1} s}$, which was interpreted in Ref. [46] as a direct result of the structure of a network of geodesics.

Relations of the type $\min [\delta D] \sim\left(L_{p}^{2} D\right)^{(1 / 3)}$, which are formally analogous to Eq. (22), were encountered in the analysis of maximum tightness achievable for a geodesics network reported in Ref. [49] and in the analysis of measur- ability of distances reported in Ref. [26]. Although once again the definitions of "min" and " $\delta D$ " used in these studies are completely different from the ones relevant for the " $\min [\delta D]$ '" of Eq. (22), the analyses reported in Refs. $[26,49]$ do provide additional motivation for the scenario (22), at least in as much as they give examples of the fact that behavior of the type $L_{p}^{2 / 3}$ can naturally emerge in quantum-gravity measurement analyses.

\section{E. Other interferometry-based quantum-gravity studies}

Several authors have put forward ideas which combine in one or another way some aspects of interferometry and candidate quantum-gravity phenomena. While the viewpoints and the results of all of these works are significantly different from the ones of the present article, it seems appropriate to at least mention briefly these studies, for the benefit of the interested reader.

A first example, on which I shall return in the next section, is provided by the idea [3] that we might be able to use modern gravity-wave interferometers to investigate certain candidate early-universe string theory effects.

The studies reported in Ref. [36] (and references therein) have considered how certain effectively stochastic properties of space-time would affect the evolution of quantummechanical states. The stochastic properties there considered are different from the ones discussed in the present article, but were introduced within a similar viewpoint, i.e., stochastic processes as effective description of quantum space-time processes. The implications of these stochastic properties for the evolution of quantum-mechanical states were modeled via the formalism of "primary state diffusion," but only rather crude models turned out to be treatable. Atom interferometers were found to have properties suitable for tests of this scenario. I should however emphasize that in Ref. [36] the proposed tests concerned the quantum mechanics of systems living in a fuzzy space-time, whereas here and in Ref. [8] I have discussed direct tests of effectively stochastic properties of space-time.

The studies reported in Refs. [34,35] are more closely related to the physics of gravity-wave interferometers. In particular, combining a detailed analysis of certain aspects of interferometry and the assumption that quantum space-time effects could be estimated using ordinary quantum mechanics applied to Einstein's gravity, Refs. [34,35] developed a model of quantum-gravity induced noise for interferometers which fits within the scenario I here discussed in Sec. II B. [Actually, Refs. [34,35] discuss in greater detail the spectral features encoded in Eqs. (3),(4), while, as explained in Sec. II B, it was for me sufficient to provide a simplified discussion.] As mentioned in Sec. II B, it is not surprising that the assumption that quantum gravity be given by an ordinary quantum mechanics applied to (some formulation of) Einstein's gravity would lead to noise levels of the type encoded in Eqs. (3),(4).

The recent paper Ref. [62] proposed certain quantum properties of gravity waves and discussed the implications for gravity-wave interferometry. Let me emphasize that instead the effects considered here and in Ref. [8] concern the 
properties of the interferometer and would affect the operation of any interferometer whether or not it would be used to detect gravity waves. Here and in Ref. [8] the emphasis on modern gravity-wave interferometers is only due to the fact that these interferometers, because of the extraordinary challenges posed by the detection of classical gravity waves, are the most advanced interferometers available and therefore provide the best opportunity to test scenarios for quantumgravity induced noise in interferometers.

\section{A QUANTUM-GRAVITY PHENOMENOLOGY PROGRAM}

While opportunities to test experimentally the nature of the interplay between gravitation and quantum mechanics remain extremely rare, the proposals now available [2-6,8] represent a significant step forward with respect to the expectations of not many years ago. We have finally at least reached the point that the most optimistic estimates of quantum-gravity effects can be falsified. In searching for even more opportunities to test quantum gravity it is useful to analyze the proposals put forward in Refs. $[2-6,8]$ as representatives of the two generic mechanisms that one might imagine to use in quantum-gravity experiments. Let me comment here on these mechanisms. The most natural discovery strategy would of course resort to strong quantum-gravity effects, of the type we expect for collisions of elementary particles endowed with momenta of order the Planck mass $\left(10^{19} \mathrm{GeV}\right)$. Since presently and for the foreseeable future we do not expect to be able to set up such collisions, the only opportunities to find evidence of strong quantum-gravity effects should be found in natural phenomena (e.g., astrophysical contexts that might excite strong quantum-gravity effects) rather than in controlled laboratory setups. An example is provided by the experiment proposed in Ref. [3] which would be looking for residual traces of some strong quantum-gravity effects (specifically, critical superstring theory effects [63]) which might have occurred in the early Universe.

Another class of quantum gravity experiments is based on physical contexts in which small quantum-gravity effects lead to observably large signatures thanks to the interplay with a naturally large number present in such contexts. This is the basic mechanism underlying all the proposals in Refs. [2,4-6] and underlying the interferometric studies of spacetime fuzziness proposed in Ref. [8] which I have here discussed in detail. For the interferometric studies which I am proposing the large number is essentially provided by the ratio between the inverse of the Planck time and the typical frequencies of operation of gravity-wave interferometers. In practice if some of the space-time fuzziness scenarios discussed in Sec. II capture actual features of quantum spacetime, in a time as long as the inverse of the typical interferometer frequency of operation (e.g., for the modern interferometers used to detect gravity waves) an extremely large number of minute quantum fluctuations in the distance $D$ could add up. A large sum of small quantities can give a sizeable final result. This is important in developing an intuition for the mechanism that allows a fuzzy picture of spacetime on scales of order $10^{-35} \mathrm{~m}\left(L_{p}\right.$ fluctuations occurring at a $1 / t_{p}$ rate) to be tested using a detector with nominal $10^{-18} \mathrm{~m}$ sensitivity (see comments above on the sensitivity of modern interferometers). The break down of the classical space-time picture occurs on distance scales of order $10^{-35} \mathrm{~m}$, but the nature of this breaking (in models such as the random-walk model of fuzziness) is such that an interefometer working at a few hundred $\mathrm{Hz}$ is effectively sensitive to a collective effect of a very large numer of minute fluctuations.

For the physical context of the gamma rays reaching us from far away astrophysical objects the large number can be provided by the ratio between the time travelled by the gamma rays and the time scale over which the signal presents significant structure (time spread of peaks, etc.). The proposal made in Ref. [4] basically uses the fact that this allows to add up a very large number of very minute dispersion-inducing quantum-gravity effects, and if the deformation of the dispersion relation goes linearly with the Planck length the resulting energy-dependent time delay turns out to be comparable to the time scale that characterizes some of these astrophysical signals.

Similarly, experiments investigating the quantum phases induced by large gravitational fields [5-7] (the only aspect of the interplay between gravitation and quantum mechanics on which we already have positive "discovery" data [6,7]) exploit the fact that gravitational forces are additive and therefore, for example, gravitational effects due to the Earth are the result of a very large number of very minute gravitational effects (instead we would not be able to measure the quantum phases induced by a single elementary particle).

The large number involved in the possibility that quantum-gravity effects might leave an observable trace $[2,39,40,64-68]$ in some aspects of the phenomenology of the neutral-kaon system cannot be directly interpreted as the number of minute quantum-gravity effects to which the system is exposed. It is rather that the conjectured quantumgravity effects would also involve, in addition to the small dimensionless ratio between the energy of the kaons and the Planck energy, a very large dimensionless ratio [2,9] characterizing the ordinary (non-quantum-gravitational) physics of neutral kaons.

The idea of finding ways to put together many minute effects (which until a short time ago had been ignored by the quantum-gravity community) has a time honored tradition in physics. Perhaps the clearest example is the particle-physics experiment setting bounds on proton lifetime. The relevant dimensionless ratio characterizing proton-decay analyses is extremely small (somewhere in the neighborhood of $10^{-64}$, since it is given by the fourth power of the ratio between the mass of the proton and the grandunification scale), but by keeping under observation a correspondingly large number of protons experimentalists are managing ${ }^{19}$ to set highly significant bounds.

\footnotetext{
${ }^{19}$ This author's familiarity [69] with the accomplishments of proton-decay experiments has certainly contributed to the moderate optimism for the outlook of quantum-gravity phenomenology which is implicit in the present article.
} 
Another point of contact between proposed quantumgravity experiments and proton-decay experiments is that a crucial role in rendering the experiment viable is the fact that the process under investigation would violate some of the symmetries of ordinary physics. This plays a central role in the experiments proposed in Refs. [2,4].

\section{MORE ON A LOW-ENERGY EFFECTIVE THEORY OF QUANTUM GRAVITY}

While the primary emphasis has been on direct experimental tests of crude scenarios for space-time fuzziness, part of this article has been devoted to the discussion (expanding on what was reported in Refs. $[10,12])$ of the properties that one could demand of a theory suitable for a first stage of partial unification of gravity and quantum mechanics. This first stage of partial unification would be a low-energy effective theory capturing only some rough features of quantum gravity, possibly associated with the structure of the nontrivial "quantum-gravity vacuum."

One of the features that appear desirable for an effective low-energy theory of quantum gravity is that its mechanics be not exactly given by ordinary quantum mechanics. I have reviewed some of the arguments $[10,12]$ in support of this hypothesis when I discussed the Salecker-Wigner setup for the measurement of distances, and showed that the problems associated with the infinite-mass classical-device limit provide encouragement for the idea that the analysis of quantum-gravity experiments should be fundamentally different from the one of the experiments described by ordinary quantum mechanics. A similar conclusion was already drawn in the context of attempts (see, e.g., Ref. [15]) to generalize to the study of the measurability of gravitational fields the famous Bohr-Rosenfeld analysis [48] of the measurability of the electromagnetic field. In fact, in order to achieve the accuracy allowed by the formalism of ordinary quantum mechanics, the Bohr-Rosenfeld measurement procedure resorts to ideal test particles of infinite mass, which would of course not be admissible probes in a gravitational context [15]. Since all of the (extensive) experimental evidence for ordinary quantum mechanics comes from experiments in which the behavior of the devices can be meaningfully approximated as classical, and moreover it is well understood that the conceptual structure of ordinary quantum mechanics makes it only acceptable as the theoretical framework for the description of the outcomes of this specific type of experiments, it seems reasonable to explore the possibility that quantum gravity might require a new mechanics, not exactly given by ordinary quantum mechanics and probably involving a novel (in a sense, "more democratic") relationship between "measuring apparatus", and "system.',

Other (related) plausible features of the correct effective low-energy theory of quantum gravity are novel bounds on the measurability of distances. This appears to be an inevitable consequence of relinquishing the idealized methods of measurement analysis that rely on the artifacts of the infinitemass classical-device limit. If indeed one of these novel measurability bounds holds in the physical world, and if indeed the structure of the quantum-gravity vacuum is nontrivial and involves space-time fuzziness, it appears also plausible that these two features be related, i.e., that the fuzziness of space-time would be ultimately responsible for the measurability bounds.

The intuition emerging from these first investigations of the properties of a low-energy effective quantum gravity might or might not turn out to be accurate, but additional work on this first stage of partial unification of gravity and quantum mechanics is anyway well motivated in light of the huge gap between the Planck regime and the physical regimes ordinarily accessed in present-day particle physics or gravity experiments. Results on a low-energy effective quantum gravity might provide a perspective on quantum gravity that is complementary with respect to the one emerging from approaches based on proposals for a one-step full unification of gravity and quantum mechanics. On one side of this complementarity there are the attempts to find a low-energy effective quantum gravity which are necessarily driven by intuition based on direct extrapolation from known physical regimes; they are therefore rather close to the phenomenological realm but they are confronted by huge difficulties when trying to incorporate the physical intuition within a completely new formalism. On the other side there are the attempts of one-step full unification of gravity and quantum mechanics, which usually start from some intuition concerning the appropriate formalism (e.g., "canonical or loop quantum gravity" [56] or "critical superstring theory"' $[32,70]$ ) but are confronted by huge difficulties when trying to "come down" to the level of phenomenological predictions. These complementary perspectives might meet at the midway point ${ }^{20}$ leading to new insight in quantum-gravity physics. One instance in which this midway-point meeting has already been successful is provided by the candidate phenomenon of quantum-gravity-induced Planck-length-linear deformation of dispersion relations, which was proposed within some phenomenological analyses $[4,19]$ of the type needed for the search of a low-energy theory of quantum gravity, but was then shown [20] to be consistent with the structure of canonical-loop quantum gravity.

\section{OUTLOOK}

The panorama of opportunities for quantum-gravity phenomenology is certainly becoming richer. In this article I have taken the conservative viewpoint that the length scales parametrizing proposed quantum-gravity phenomena should be somewhere in the neighborhood of the Planck length, but I have taken the optimistic (although supported by various quantum-gravity scenarios, including canonical or loop quantum gravity [20,56]) viewpoint that there might be quantum-gravity effects going linearly or quadratically with the Planck length, i.e., effects which are penalized only by one or two powers of the Planck length.

\footnotetext{
${ }^{20}$ Among the tools available for the exploration of this possible "middle ground" another significant example is provided by the approach of Ref. [71], which is based on an effective low-energy quantum-gravity action.
} 
An exciting recent development is that results in the general area of string theory have motivated work (see, e.g., Ref. [72]) on theories with large extra dimensions in which rather naturally quantum-gravity effects would become significant at scales much larger than the conventional Planck length. In such scenarios one expects to find phenomena for which the length scale characterizing the onset of large quantumgravity corrections is much larger than the conventional Planck length.

The example of advanced modern interferometers here considered provides further evidence (in addition to the one emerging from Refs. $[39,4]$ ) of the fact that we might have a chance to find signatures of quantum-gravity effects if they are linear in the conventional Planck length. [For the example of modern interferometers I have shown that sensitivity to $L_{p}$-linear quantum-gravity-induced $\sigma_{L}^{2}$ has already been achieved.] If Nature only hosts effects that are quadratic in the deformation length scale, values of this length scale of order the Planck length might be out of reach for a long time, but effects quadratic in the larger length scales characterizing scenarios of the type in Ref. [72] should be experimentally accessible.

On the theory side an exciting opportunity for future research appears to be provided by the possibility of exchanges of ideas between the more phenomenological or intuitive studies appropriate for the search of a low-energy effective quantum gravity and the more rigorous or formal studies used in searches of fully consistent quantum-gravity theories. As mentioned at the end of the preceding section, the first example of such an exchange has led to the exciting realiza- tion that deformed dispersion relations linear in the Planck length appear plausible $[4,12,19]$ from the point of view of heuristic phenomenological analyses and are also consistent [20] with the structure of canonical or loop quantum gravity. Additional exchanges of this type appear likely. For example, the intuition coming from the low-energy effective quantum-gravity viewpoint on distance fuzziness which I discussed here might prove useful for those quantum-gravity approaches (again an example is provided by canonical or loop quantum gravity) in which there is substantial evidence of space-time fuzziness but one has not yet achieved a satisfactory description of fuzzy distances.

\section{ACKNOWLEDGMENTS}

I owe special thanks to Abhay Ashtekar, since he suggested to me that gravity-wave interferometers might be useful for experimental tests of some of the quantum-gravity phenomena that I have been investigating. My understanding of Refs. [39] and [3] benefited from conversations with N.E. Mavromatos and G. Veneziano. I am also happy to acknowledge a correspondence from A. Camacho which provided positive feedback on my Ref. [8] and also made me aware of the works in Refs. [34,35,62]. Still on the "theory side" I am grateful to several colleagues who provided encouragement and stimulating feedback, particularly D. Ahluwalia, J. Ellis, J. Lukierski, C. Rovelli, S. Sarkar, L. Smolin and J. Stachel. On the "experiment side" I would like to thank F. Barone, J. Faist, R. Flaminio, L. Gammaitoni, T. Huffman, L. Marrucci and M. Punturo for useful conversations on various aspects of interferometry.
[1] C.J. Isham, "Structural issues in quantum gravity," in Proceedings of General Relativity and Gravitation, Florence, 1995 (to be published).

[2] J. Ellis, J.S. Hagelin, D.V. Nanopoulos, and M. Srednicki, Nucl. Phys. B241, 381 (1984).

[3] R. Brustein, M. Gasperini, M. Giovannini, and G. Veneziano, Phys. Lett. B 361, 45 (1995).

[4] G. Amelino-Camelia, J. Ellis, N.E. Mavromatos, D.V. Nanopoulos, and S. Sarkar, Nature (London) 393, 763 (1998).

[5] D.V. Ahluwalia, Mod. Phys. Lett. A 13, 1393 (1998).

[6] R. Colella, A.W. Overhauser, and S.A. Werner, Phys. Rev. Lett. 34, 1472 (1975).

[7] B. Young, M. Kasevich, and S. Chu, Atom Interferometry (Academic, New York, 1997).

[8] G. Amelino-Camelia, Nature (London) 398, 216 (1999).

[9] G. Amelino-Camelia, CERN-TH/99-223, "'Are we at the dawn of quantum-gravity phenomenology?," notes based on lectures given at the XXXV Karpacz Winter School of Theoretical Physics, From Cosmology to Quantum Gravity, Polanica, Poland, 1999 (to appear in the proceedings).

[10] G. Amelino-Camelia, Mod. Phys. Lett. A 9, 3415 (1994); 11, 1411 (1996).

[11] G. Amelino-Camelia, Phys. Lett. B 392, 283 (1997).

[12] G. Amelino-Camelia, Mod. Phys. Lett. A 13, 1319 (1998).
[13] J.A. Wheeler, in Relativity, Groups and Topology, edited by B.S. and C.M. De Witt (Gordon and Breach, New York, 1963).

[14] S.W. Hawking, Nucl. Phys. B144, 349 (1978); Phys. Rev. D 18, 1747 (1978); S.W. Hawking, D.N. Page, and C.N. Pope, Nucl. Phys. B170, 283 (1980).

[15] P.G. Bergmann and G.J. Smith, Gen. Relativ. Gravit. 14, 1131 (1982).

[16] E.P. Wigner, Rev. Mod. Phys. 29, 255 (1957); H. Salecker and E.P. Wigner, Phys. Rev. 109, 571 (1958).

[17] A. Ashtekar, C. Rovelli, and L. Smolin, Phys. Rev. Lett. 69, 237 (1992).

[18] J. Ellis, N. Mavromatos, and D.V. Nanopoulos, Phys. Lett. B 293, 37 (1992).

[19] G. Amelino-Camelia, J. Ellis, N.E. Mavromatos, and D.V. Nanopoulos, Int. J. Mod. Phys. A 12, 607 (1997).

[20] R. Gambini and J. Pullin, Phys. Rev. D 59, 124021 (1999).

[21] P.R. Saulson, Fundamentals of Interferometric Gravitational Wave Detectors (World Scientific, Singapore, 1994).

[22] A. Abramovici et al., Phys. Lett. A 218, 157 (1996).

[23] A. Abramovici et al., Science 256, 325 (1992).

[24] C. Bradaschia et al., Nucl. Instrum. Methods Phys. Res. A 289, 518 (1990); B. Caron et al., Class. Quantum Grav. 14, 1461 (1997).

[25] K. Danzmann, Class. Quantum Grav. 13, A247 (1996). 
[26] Y.J. Ng and H. Van Dam, Mod. Phys. Lett. A 9, 335 (1994); 10, 2801 (1995).

[27] V.B. Barginskii and L.P. Grishchuk, Zh. Éksp. Teor. Fiz. 89, 744 (1985) [Sov. Phys. JETP 62, 427 (1985)]; R. Brustein, gr-qc/9810063.

[28] B.S. De Witt, in Gravitation, An Introduction to Current Research, edited by L. Witten (Wiley, New York, 1962).

[29] T. Padmanabhan, Class. Quantum Grav. 4, L107 (1987).

[30] See, e.g., L.J. Garay, Int. J. Mod. Phys. A 10, 145 (1995).

[31] G. Veneziano, Europhys. Lett. 2, 199 (1986); D.J. Gross and P.F. Mende, Nucl. Phys. B303, 407 (1988); D. Amati, M. Ciafaloni, and G. Veneziano, Phys. Lett. B 216, 41 (1989); K. Konishi, G. Paffuti, and P. Provero, ibid. 234, 276 (1990); T. Yoneya, Mod. Phys. Lett. A 4, 1587 (1989).

[32] J. Polchinski, Superstring Theory and Beyond (Cambridge University Press, Cambridge, England, 1998).

[33] D. Kabat and P. Pouliot, Phys. Rev. Lett. 77, 1004 (1996); M.R. Douglas, D. Kabat, P. Pouliot, and S.H. Shenker, Nucl. Phys. B485, 85 (1997).

[34] M.-T. Jaekel and S. Reynaud, Europhys. Lett. 13, 301 (1990).

[35] M.-T. Jaekel and S. Reynaud, Phys. Lett. B 185, 143 (1994).

[36] I.C. Percival and W.T. Strunz, Proc. R. Soc. London A453, 431 (1997).

[37] L.J. Garay, Phys. Rev. Lett. 80, 2508 (1998).

[38] V. Radeka, IEEE Trans. Nucl. Sci. NS16, 17 (1969); Annu. Rev. Nucl. Part. Sci. 38, 217 (1988).

[39] J. Ellis, J. Lopez, N. Mavromatos, D. Nanopoulos, and CPLEAR Collaboration, Phys. Lett. B 364, 239 (1995).

[40] P. Huet and M.E. Peskin, Nucl. Phys. B434, 3 (1995).

[41] S.W. Hawking and S.F. Ross, Phys. Rev. D 56, 6403 (1997).

[42] I.L. Egusquiza, L.J. Garay, and J.M. Raya, quant-ph/9811009.

[43] R.J. Adler, I.M. Nemenman, J.M. Overduin, and D.I. Santiago, gr-qc/9909017.

[44] G. Amelino-Camelia, Phys. Lett. B 477, 436 (2000).

[45] J. Ellis, N.E. Mavromatos, and D.V. Nanopoulos, Phys. Rev. D 61, 027503 (2000); A. Campbell-Smith, J. Ellis, N.E. Mavromatos, and D.V. Nanopoulos, Phys. Lett. B 466, 11 (1999).

[46] L. Diosi and B. Lukacs, Phys. Lett. A 142, 331 (1989).

[47] D.V. Ahluwalia, Phys. Lett. B 339, 301 (1994).

[48] N. Bohr and L. Rosenfeld, K. Dan. Vidensk. Selsk. Mat. Fys. Medd. 12, 1 (1933).

[49] F. Karolyhazy, Nuovo Cimento A 42, 390 (1966).

[50] C. Rovelli, Class. Quantum Grav. 8, 297 (1991); 8, 317 (1991).

[51] J. Lukierski, A. Nowicki, and H. Ruegg, Ann. Phys. (N.Y.) 243, 90 (1995).
[52] G. Amelino-Camelia, J. Lukierski, and A. Nowicki, Int. J. Mod. Phys. A 14, 4575 (1999).

[53] G. 't Hooft, Class. Quantum Grav. 13, 1023 (1996).

[54] W. Pauli, in Die allgemeinen prinzipien der Wallen-mechanik. Handbuch der Physik, edited by S. Fluegge (Springer, Berlin, 1958).

[55] P. Astone et al., Phys. Lett. B 385, 421 (1996).

[56] A. Ashtekar, Phys. Rev. Lett. 57, 2244 (1986); C. Rovelli and L. Smolin, ibid. 61, 1155 (1988).

[57] F. Markopoulou, gr-qc/9704013; F. Markopoulou and L. Smolin, Phys. Rev. D 58, 084033 (1998).

[58] S. Majid and H. Ruegg, Phys. Lett. B 334, 348 (1998).

[59] A. Nowicki, E. Sorace, and M. Tarlini, Phys. Lett. B 302, 419 (1993).

[60] S. Doplicher, K. Fredenhagen, and J.E. Roberts, Phys. Lett. B 331, 39 (1994); Commun. Math. Phys. 172, 187 (1995); S. Doplicher, Ann. Inst. Henri Poincaré: Phys. Theor. 64, 543 (1996).

[61] A. Kempf, J. Math. Phys. 35, 4483 (1994); A. Kempf, G. Mangano, and R.B. Mann, Phys. Rev. D 52, 1108 (1995); A. Kempf and G. Mangano, ibid. 55, 7909 (1997).

[62] A. Camacho, Int. J. Mod. Phys. A 14, 1997 (1999).

[63] G. Veneziano, Phys. Lett. B 265, 287 (1991); M. Gasperini and G. Veneziano, Astropart. Phys. 1, 317 (1993).

[64] V.A. Kostelecky and R. Potting, Phys. Rev. D 51, 3923 (1995).

[65] J. Scherk, Phys. Lett. 88B, 265 (1978); J. Scherk, in Proceedings of the 1979 Supergravity Workshop at Stony Brook, edited by P. van Nieuwenhuizen and D.Z. Freedman (North-Holland, Amsterdam, 1979).

[66] S. Bellucci and V. Faraoni, Phys. Rev. D 49, 2922 (1994); Phys. Lett. B 377, 55 (1996).

[67] F. Benatti and R. Floreanini, Nucl. Phys. B488, 335 (1997).

[68] G. Amelino-Camelia and F. Buccella, CERN-TH/2000-034 (in preparation).

[69] G. Amelino-Camelia, Laurea thesis, Facoltá di Fisica dell'Universitá di Napoli, 1990.

[70] M.B. Green, J.H. Schwarz, and E. Witten, Superstring Theory (Cambridge University Press, Cambridge, England, 1987).

[71] J.F. Donoghue, Phys. Rev. Lett. 72, 2996 (1994); Phys. Rev. D 50, 3874 (1994); A. Akhundov, S. Bellucci, and A. Shiekh, Phys. Lett. B 395, 16 (1997).

[72] I. Antoniadis, Phys. Lett. B 246, 377 (1990); J. Lykken, Phys. Rev. D 54, 3693 (1996); N. Arkani-Hamed, S. Dimopoulos, and G. Dvali, Phys. Lett. B 429, 263 (1998); K.R. Dienes, E. Dudas, and T. Gherghetta, ibid. 436, 55 (1998); P.C. Argyres, S. Dimopoulos, and J. March-Russell, ibid. 441, 96 (1998). 